\title{
Genomic and RNA-Seq Profiling of Patients with Heart Failure Identified Alterations in Phosphorylation and Immune Signaling Pathways
}

\section{Shikuan Zhang}

Beijing Institute of Heart, Lung and Blood Vessel Disease, Capital Medical University

\section{Xin Li}

Medical Innovation Research Division of Chinese PLA General Hospital

\section{Xiang Xu}

Medical Innovation Research Division of Chinese PLA General Hospital

\section{Qingxia Wei}

Medical Innovation Research Division of Chinese PLA General Hospital

\section{Hanlu Li}

Medical Innovation Research Division of Chinese PLA General Hospital

\section{Tong Liu}

Beijing Institute of Heart, Lung and Blood Vessel Disease, Capital Medical University

Chunlei L. Liu ( $\square$ chunleiliu87@163.com )

Medical Innovation Research Division of Chinese PLA General Hospital

\section{Research Article}

Keywords: heart failure, immune signaling, phosphorylation, RNA-Seq

Posted Date: August 17th, 2021

DOl: https://doi.org/10.21203/rs.3.rs-625496/v1

License: (c) (1) This work is licensed under a Creative Commons Attribution 4.0 International License. Read Full License 


\section{Abstract}

Background: Heart failure (HF) is a complex pathophysiological state in which the delivery of blood and nutrients to the tissues is inadequate. It is rarely curable and often associated with poor prognosis. The current study aimed to analyze the exomic and RNA-Seq data of patients with HF to identify the key altered pathways.

Methods: Heart failure participants were recruited, and whole blood samples were collected. The samples were used for whole exome sequencing and RNA-Seq analysis. Gene expression and RNA-Seq results were verified by Gene chips and RT-PCR

Results: We report a dysregulation of phosphorylation and immune signaling in patients with HF both by exomic and RNA-Seq data. We identified mutations in TITIN,OBSCURIN, NOD2, CDH2, MAP3K5, and SLC17A4 to be associated with HF by exomic analysis. And certain genes, S100A12, S100A8, S100A9, PFDN5, and TMCC2, were found upregulated in patients with HF by RNA-Seq.

Conlcusion: Our results demonstrated the overall disruption of key phosphorylation and immune signaling pathways in patients with HF.

\section{Background}

Heart failure (HF) is a major public health problem, affecting more than 4.5 million individuals in China. More than 23 million new cases are diagnosed every year worldwide, with > 550,000 new cases in China [1]. The survival estimates following diagnosis are $50 \%$ and $10 \%$ for 5 and 10 years, respectively. HF defined as a disease of myocardium characterised by systolic dysfunction, either of the left ventricle (LV)

alone or biventricular and LV dilatation that cannotbe explained exclusively by abnormal loading or coronary artery disease. In patients with Left ventricular dysfunction is considered to be particularly associated with the increased risk of sudden death $[2,3]$. Coronary heart disease and hypertension are the major underlying causes of HF [4]. Racial differences also influence the occurrence and outcome of HF, and heritability estimates of variability in the left ventricular mass have been found to be $28-65 \%$, hence, suggesting a genetic contribution to the pathophysiology of left ventricular remodeling and HF [5]. However, the reason underlying the higher risk of HF in some patients with these conditions is unclear and deciphering critical mediators from nonspecific factors is often difficult in the context of underlying heart diseases.

Mutation-induced HF has been linked primarily to polymorphisms in several sarcomeric genes, such as those encoding cardiac troponin T2, cardiac a-actin, titin, cardiac-myosin binding protein $\mathrm{C}$, and betamyosin heavy chain ( $\beta-\mathrm{MHC}$ ) [6]. Targeted sequencing of dilated cardiomyopathy (DCM)-related genes in patients with HF has revealed mutations in MYH7, TNNT2, TTN, LMNA, RBM20, and SCN5A and that of hypertrophic cardiomyopathy (HCM)-related genes has revealed mutations in $M Y H, M Y B P C 3, T N N T 2$, TNNT3, TPM1, MYL2, MYL3, ACTC1, PLN, FLNC, GLA, LAMP2, PPKAG2, and TTR, as per reports by both 
Chinese and American scholar from Heart Association [7, 8]. These mutations result in aberrant proteins in the sarcomere, which in turn affect the contractile function of the latter; this induces cardiac remodeling through dysregulated downstream signaling pathways, including calcium signaling, G-protein, protein kinase $C$ (PKC), and renin-angiotensin system/ mitogen-activated protein kinase (MAPK) pathways $[9,10]$. Pro-inflammatory cytokines and immune-related pathways have also been studied in relation to HF [11]. Tumor necrosis factor (TNF) is upregulated in patients with severe congestive heart failure [12]. The roles of interleukin, endothelin, and other cytokines are still under investigation, and their upregulation has been proven to be detrimental under certain conditions [13].

Several therapeutics that target HF are being evaluated currently. Our efforts in this study focused on a cross-platform integrated analysis of patients with HF, focusing on the dissection of molecular pathogenesis. In this study, we comprehensively used whole exome sequencing, gene chip, and RNA-Seq technology to find the gene expression changes in patients with HF.

\section{Methods}

\section{Human participants and sampling}

For human studies, all participants were recruited, and whole blood samples were collected correspondingly from PLA general hospital. The blood samples were kept at BD paxgene tube for RNA extraction and kept on BD EDTA routine blood tube for DNA and plasma extraction. The diagnosis of HF was based on standard guidelines according to the symptoms or signs, electrocardiogram, chest radiograph, and echocardiography. Patients were included in the sequencing if they had a reduced ejection fraction $(\mathrm{LVEF}<40 \%)$.

\section{Exome sequencing library preparations and analysis}

Genomic DNA was extracted from Human whole bloods. Clearseq inherited disease capture kit were used to capture. $10 \times$ of Whole-exome sequencing data were covered. The raw reads were filtered to high quality clean data and then were used to align against the human reference genome by Burrows-Wheeler Aligner. Alignment files were converted to BAM files and then translated to SNP. ANNOVAR was used to analysis.

\section{Gene chips}

Gene chips containing cardiovascular diseases-related genes were used in this study. 1 ug of genomic DNA was fragmented and hybridized to the capture probes following the manufacturer's protocols (Roche). The resulting libraries were sequenced on the Illumina Hiseq 2000 platform to generate pairedend reads of $90 \mathrm{bp}$. Finally, a mean coverage of $153.01 \times$ for 957 samples was got.

\section{Multiplex immunoassay}


Venous blood samples $(20 \mathrm{~mL})$ were collected in EDTA Routine blood tubes (BD, Franklin Lake, NJ, USA) and immediately centrifuged at 3,000 rpm for $15 \mathrm{~min}$. Plasma samples were aliquoted and stored at -80 ${ }^{\circ} \mathrm{C}$. The samples were frozen and thawed only once. We used a human cytokine and chemokine Bio-Plex assay kit (BioRad Laboratories, Milan, Italy), bead based multiplex immunoassay. The factors analyzed were S100A12; APRIL TNFSF13; BAFF TNFSF13B; Chitinase 3-like 1; IFN-a2; IFN-b; IFN-r; IL-10; IL-11; IL12(p40); IL-12(p70); IL-19; IL-2; IL-20; IL-22; IL-26; IL-27(p28); IL-28A IFN-labuda2; IL-29; IFN-labuda1; IL-32; IL-34; IL-35; IL-8; LIGHT TNFSF14; MMP-1; MMP-2; MMP-3; Osteocalcin; Osteopontin; Pentraxin-3; TSLP; TWEAK TNFSF12; gp130 sIL-6Rb; sCD163; sCD30; TNFRSF8; sIL-6Ra; sTNF-R1;sTNF-R2;Ckine; BCA-1; CTACK; ENA-78; Eotaxin; Eotaxin-2; Eotaxin-3; Fractalkine; GCP-2/CXCL6; GM-CSF; Gro-a; Gro-b/CXCL2; I309; IFN-r; IL-1b; IL-2; IL-4; IL-6; IL-8; IL-10; IL-16; IP-10; I-TAC; MCP-1; MCP-2; MCP-3; MCP-4; MDC; MIF; MIG; MIP-1a; MIP-1d; MIP-3a; MIP-3B; MPIF-1; SCYB16; SDF-1a + B; TARC; TECK; TNF-a. The plasma concentrations of these molecules were tested for all rats used in the study. The plasma samples were diluted 1:4 and tested in duplicate. The results were determined using a Bio-Plex 200 instrument equipped with Bio-plex Manager software (version 4.1; Bio-Rad Laboratories), using a five-parameter non-linear regression formula to compute the sample concentrations from standard curves.

\section{RNA-sequencing}

Human bloods were stored in BD paxgene tube. Total RNA was extracted using the RNeasy Mini Kit (Qiagen, Dusseldorf, Germany) and RNA concentration was measured using NanoDrop 2000 (Thermo). RNA Nano 6000 Assay Kit was used to measure RNA integrity. NEB Next UltraTM RNA Library Prep Kit was used to generate sequencing library. And then the library preparated was sequenced on Illumina Hiseq X Ten platform. Clean reads transformed from Raw sequences were mapped to human genome reference. Only reads with a perfect match were then for further analysis. Differential expression analysis was performed using the DESeq $R$ package (1.10.1). The resulting $P$ values were adjusted using the Benjamini controlled to the false discovery rate. P-value $<0.01$ were assigned as differentially expression.

\section{RT-PCR}

Tissues were lysed using QIAzol Lysis Reagent (Qiagen, Hilden, Germany) and total RNA was extracted using a RNeasy Mini Kit (Qiagen), according to the manufacturer's protocols. RNA quality was assessed using an Bioanalyzer Nano RNA Chip (Agilent, Santa Clara, CA, USA) based on the 260/280 nm absorbance ratio of 1.8-2.0. High-quality RNA was then reverse-transcribed to cDNA with SuperScript III (Invitrogen, Waltham, MA, USA), and mRNA expression was assessed using the cDNAs as templates with SYBR Green and the following primers (shown in the 5'-3' orientation): S100A12 (forward:

ATACTCAGTTCGGAAGGGGC, reverse: TTTGTGGGTGTGGTAATGGG); S100A8 (forward: TCATCGACGTCTACCACAAGT, reverse: CTGCCACGCCCATCTTTATC); S100A9 (forward: CAGCTGGAACGCAACATAGA, reverse: CTGCATTTGTGTCCAGGTCC); LGALS3 (forward: AACTGGGGAAGGGAAGAAAGAC, reverse: GAAGCACTGGTGAGGTCTATGT); CISD2 (forward: CGCTAGGCTCACAGTTTCAGA, reverse: ACGCCAACACCTACAATAAGC); TRAPPC5 (forward: GCGCACCTTCTACATCATCG, reverse: GATGACTGCCTCCTCGAACT); PDCD10 (forward: 
AGTCCCTTCTTCGTATGGCA, reverse: TGATTGTCTGCAGAAACCTCA); PFDN5 (forward:

GTTCTTGTCCACGTCCATTGC, reverse: TCTTTCCCCTCGTTGCTCTTG); TMCC (forward:

AACAACAGGGCGAGGAGGAT, reverse: CAAACATGGAGCCCTCTGACA).

\section{Protein chip analysis}

The samples were analyzed with a Human Cytokine G1000 arrays (AAH-CYT-G1000; ayBiotech, Norcross, GA, USA) according to the manufacturer's instructions. Briefly, each sample was biotinylated and added onto duplicate glass slides preprinted with capture antibodies. Bound proteins were incubated with a streptavidin-conjugated fluorescent dye and the observed fluorescence intensities were normalized to the intensities of the internal positive controls. The arrays were scanned using an excitation wavelength of $532 \mathrm{~nm}$, and the fluorescence was measured using an InnoScan 300 Microarray Scanner.Differentially expressed proteins were arranged using hierarchical clustering and represented as a heat map.

\section{Data analysis and statistics}

Statistics analysis was performed using the SPSS 20.0 software, and the nonparametric test and twosided X 2 -test were used to compare the differences between the two groups. A P-value of $<0.05$ was considered statistically significant.

\section{Results}

\section{Whole exome sequencing results and validation}

We analyzed 17 human whole blood samples, including seven from patients volunteers diagnosed with HF with a mean ejection fraction (EF) of $34 \%$ and 10 from healthy volunteers as controls (CNTs), using whole exome sequencing. The mean \pm SD of clinical characteristics of all he volunteers is indicated in Supplementary materials (Supplementary Table 1). We found 430 variants to be present in the proband (Supplementary Table 2). We functionally annotated two clusters of genes with altered expression levels which encoded proteins for serine/threonine kinase activity and immune response (Fig. 1). We detected 35 variants in 29 genes in the phosphatase/kinase group and 45 variants in 36 genes in the immune response group (Table 1 ).

To validate the results obtained using whole exome sequencing, we expanded the sample size. We analyzed 1,000 patients volunteers with HF and 250 healthy volunteers. The mean \pm SD of clinical characteristics of all the volunteers is indicated in Supplementary Table 3. We used a gene chip and evaluated the association between the SNPs of phosphatase/kinase genes and immune response genes. We confirmed that 13 previously reported gene loci (PLA2G2A, OBSCN, UGT1A3, TTN, DAG1, SLC17A4, MAP3K5, SMG6, CDH2, OBSCN, NOD2, ACE, and CCDC40) exhibited significant changes in expression at the genome-wide level. Maximum mutations were detected in NOD2, CDH2, MAP3K5, SLC17A4, with OBSCN and TTN being the most mutated genes (Table 2). 


\section{Inflammatory cytokine profiles in the plasma of patients with HF}

Multiplex immunoassay-based screening for cytokines was performed on the samples from patients with HF and CNTs. The inflammatory factors included S100A12, IL-10, osteopontin, sCD163, sCD30 TNFRSF8, gp130 sIL-6Ra, sTNF-R1, sTNF-R2, BCA-1, CTACK, eotaxin-2, fractalkine, IL-8, IP-10, I-TAC, MCP-1, MIG, MIP-1a, MIP-3a, MPIF-1, and TEC. Most inflammatory cytokines were upregulated in patients with HF, except for IL-10, which was downregulated. In particular, sTNF-R1, sTNF-R2, fractalkine, IL-8, MIP-1a, and MPIF-1 were significantly upregulated in patients with HF (Table 2).

\section{RNA-Seq results and validation}

To investigate the differentially expressed genes (DEGs) in patients with HF, the samples used for whole exome sequencing were subjected to RNA-Seq analysis. Significance analysis of the RNA-Seq results revealed 810 DEGs: 197 upregulated $(\geq 1.5$-fold, $P<0.01)$ and 613 downregulated $(\leq 1.5$-fold, $P<0.01$; Fig. 2A, Supplementary Table 4). Functional analysis of the genes was performed using the DAVID database. We performed Gene Ontology (GO) term enrichment for identifying the genes regulating functional processes with biological significance. Functional annotation of two clusters of the significantly dysregulated genes revealed enrichment of genes corresponding to protein autophosphorylation and inflammatory response (Fig. 2B).

To validate the results obtained through RNA-Seq analysis, qRT-PCR was performed with an expanded sample size. We selected nine genes from the altered phosphorylation and inflammatory response groups, namely TRAPPC5, PDCD, PFDN5, and TMCC2 from the phosphorylation group and S100A12, S100A8, S100A9, LGGALS3, and CISD2 from the inflammatory response group. qRT-PCR analysis revealed increased expression of the nine genes in the HF samples (Fig. 2C). The qPCR results, thus, validated the results of RNA-SEq. STRING analysis elucidated the relationship of the dysregulated genes with altered phosphorylation or inflammatory response pathways (Fig. 2D).

\section{Discussion}

In this study, we identified phosphorylation and inflammatory responses as the two biological pathways associated with HF, using RNA-Seq and whole exome sequencing. We also showed that mutations in patients with HF are located in TTN, OBSCN, NOD2, CDH2, MAP3K5, and SLC17A4, and the genes that showed upregulated expression in patients with HF were S100A12, S100A8, S100A9, PFDN5, and TMCC2.

Technological advances have enabled the use of whole exome sequencing for the study of human diseases. However, the cost per base of next-generation sequencing platforms still precludes the generation of large sample sizes of completely sequenced genomes with high coverage [14]. In comparison, the gene chip technology is much cheaper. Therefore, there has been considerable focus on whole exome sequencing as the first step, with gene chip as the validation step [15]. RNA-Seq is used for transcriptome profiling using deep-sequencing technologies, and it generates millions of short sequences 
in a single run. These fragments, or 'reads', can be used to measure gene expression levels and to identify novel splicing events [16]. In this study, we comprehensively used whole exome sequencing, gene chip, and RNA-Seq technology to find the gene expression changes in patients with HF. Both whole exome sequencing and RNA-Seq identified phosphorylation and inflammatory response as the two key pathways associated with HF. These results were further verified using gene chip analysis.

Many eukaryotic cell functions, including signal transduction, cell adhesion, gene transcription, RNA splicing, apoptosis, and cell proliferation, are regulated via protein phosphorylation [17]. The expression of cardiac phosphatases is increased in patients with end-stage HF [18]. Muscle contraction and its molecular motor myosin are regulated through the phosphorylation of cardiomyocyte cytoskeletal proteins, such as the regulatory myosin light chain (MLC2). Decreased levels of phosphorylated MLC2 (MLC2-P) have been observed in HF [19, 20]. HF top-down quantitative proteomics has identified the phosphorylation of cardiac troponin I (cTnl) as a candidate biomarker for chronic HF [21]. cMyBP-C phosphorylation clearly has a direct effect on the contractile properties of the heart, sarcomere organization, and its ability to attenuate the development of HF [22]. Chronic protein kinase A (PKA) hyperphosphorylation of RyR2 results in a diastolic leak that causes cardiac dysfunction [23]. The p38 MAPK pathway is a potential target in the therapeutic regimens for infarction, hypertrophy, and HF [24]. Consistent with results of these previous reports, the current study elucidated the role of altered phosphorylation in HF, using RNA sequencing and whole exome sequencing.

In addition, we demonstrated alteration of inflammation processes in HF. The mechanisms that drive the development of HF can be divided into four broad categories [25]. The first is based on traditional risk factors, such as ischemic injury, hypertension, and metabolic syndrome; the second includes genetic cardiomyopathies; the third is based on valve dysfunction. In these three categories, the initial insult is not immune-based, rather, activation of immune system is a secondary response; and the fourth category is immune-based, which includes autoimmune and infectious (viral and bacterial) triggers. Therefore, $\mathrm{HF}$ cannot occur without inflammation. Upregulation of pro-inflammatory cytokines has been implicated in the progression of HF, and elevated inflammatory markers constitute important risk factors for HF [26]. The roles of interleukin, endothelin, and other cytokines are still under investigation, and their overexpression has been indicated to be harmful under certain conditions [27]. In this study, we not only showed an inflammatory response in $\mathrm{HF}$, but also highlighted the altered expression of a series of chemokines, such as CD163, sCD30 TNFRSF8, gp130 sIL-6Ra, sTNF-R1, sTNF-R2, BCA-1, CTACK, eotaxin2, fractalkine, IL-8, IP-10, I-TAC, MCP-1, MIG, MIP-1a, MIP-3a, MPIF-1, and TECK, as well as cytokines in the plasma of patients with HF.

Gene chip verification using a bigger sample size identified that the mutations in patients with $\mathrm{HF}$ were mainly in $T T N, O B S C N, N O D 2, C D H 2, M A P 3 K 5$, and SLC17A4. The two most significantly mutated genes were TTN and OBSCN. Mutation in the TTN gene locus $2 \mathrm{q} 31$ has been implicated in human skeletal muscle and heart diseases for over 15 years [28]. However, only recently, TTN mutation was found to be associated with HF [29]. Mutations in $O B S C N$ variants are also relatively common in inherited cardiomyopathies. Unique $O B S C N$ variants have been found in a group of 30 end-stage failing hearts [30], 
wherein the frequency was similar to that for TTN-truncating mutations, which were proposed to be the major alterations associated with patients with HF. Interestingly, obscurin (encoded by OBSCN) interacts with titin (encoded by TTM) through the $\mathrm{N}$-terminal domain, which in turn interacts with M-line complexes of titin and myomesin, hence, enhancing the binding and contributing to stability [31]. In addition, titin and obscurin contain signaling domains close to their C-terminal (a protein serine/threonine kinase domain in titin, and a Rho-GTPase GTP-GDP exchange factor domain in obscurin) that can be coupled to two serine/threonine protein kinase domains [32]. Phosphorylation of a specific sequence in the cardiac isoform (N2B region) by cAMP-dependent or CGMP-dependent protein kinases results in acute reduction in stiffness [33]. Based on the gene chip analysis, we identified that nearly $70 \%$ of mutations were related to $T T N$ and nearly $40 \%$ of mutations were related to OBSCN. Therefore, targeting TTN and OBSCN as therapeutic strategies will be highly significant. The pathological increase in passive stiffness caused by TTN and $O B S C N$ mutations may be reversed by PKA and cGMP-dependent protein kinase (PKG) [34]. In addition, We also found NOD2, CDH2, MAP3K5, and SLC17A4 mutations in HF here. NOD2 plays a key role in the immune response to intracellular bacterial lipopolysaccharides [35]. $\mathrm{CDH} 2$ encodes a classical cadherin and is a member of the cadherin superfamily, generating a calcium-dependent cell adhesion molecule. The glycoprotein MAP3K5 is a member of the MAPK signaling cascade, abundantly expressed in the human heart [36]. SLC17A4 is a sodium/phosphate cotransporter in the intestinal mucosa and plays an important role in the absorption of phosphate from the intestine [37]. The significance of mutations in the genes encoding these four proteins as therapeutic targets will require further validation, as strong mutations in these genes were not identified in this study.

S100 proteins are localized in the cytoplasm and/or nucleus of a wide range of cells, and are majorly secreted from neutrophils, monocytes, and macrophages [4]. S100 proteins are recognized for their potential in inflammation [38]. S100A8/A9 is homologous to S100A12, which provides a significant predictive value for one-year mortality in elderly patients with severe HF [39]. Our group was the first to prove, in a previous study, that S100A12 is a potential biomarker for the prediction of HF. Upregulation of S100A12, S100A8, and S100A9 in patients with HF was consistent across our studies. Further, S100A8, S100A9, and S100A12 could be potential indicators in evaluating HF [40, 41]. In addition, PFDN5 and TMCC2 were upregulated in RNA-Seq, which was further verified using real-time PCR. PFDN5 encodes a member of the prefoldin alpha subunit family, and TMCC is a novel protein of the endoplasmic reticulum. Misfolded proteins are upregulated in patients with chronic HF, and misfolded PFDN5 and TMCC2 are promising biomarkers for the prediction of HF.

Our study revealed the key pathways and molecules implicated in HF; however, it has several limitations. First, the initial population used for RNA-Seq and whole exome sequencing was modest, which decreased the sensitivity and accuracy of detecting the changes in expression levels and gene variants that do not make large contributions to HF. Second, the SNP coverage was relatively low when we used a large sample to verify the variants.

In conclusion, we verified that phosphorylation and inflammation are associated with $\mathrm{HF}$, and confirmed that compounds partly inhibiting protein phosphatase and inflammation can have cardiac protective 
effects.

\section{Abbreviations}

$\mathrm{HF}$, heart failure; PP1, protein phosphatase type-1; HCM, hypertrophic cardiomyopathy; DCM, dilated cardiomyopathy; TNF, tumor necrosis factor; $E F$, ejection fraction; DEGs, differentially expressed genes; CNTs, controls; cTnl, cardiac troponin I; MAPK, mitogen-activated protein kinase; TTN, Titin; OBSCN, obscurin

\section{Declarations}

\section{Acknowledgments}

Not applicable.

\section{Funding}

This work was supported by National Key R\&D Program for Precision medicine [Grant Number 2017YFC0908400].

\section{Availability of data and materials}

All data generated or analyzed during this study are included in this published article.

\section{Authors' contributions}

SKZ conceived and designed the experiments, performed the experiments,

$\mathrm{XL}$ and $\mathrm{XX}$ analyzed the data, contributed reagents/materials/analysis tools, prepared figures and/or tables, authored or reviewed drafts of the paper, approved the final draft.

QXW and HLL analyzed the data.

SKZ and XL performed the experiments, prepared figures and/or tables, authored or reviewed drafts of the paper.

TL conceived and designed the experiments, approved the final draft.

SKZ and CLL confirm the authenticity of all the raw data.

\section{Ethics approval and consent to participate}

The study was approved by the Ethics Committee of the Chinese PLA General Hospital, and the document number is S.2017-035-01. 


\section{Patient consent for publication}

Informed consent was provided by all of the patients before participation in the study.

\section{References}

1. Roger VL. Epidemiology of Heart Failure. Circ Res. 2013,113:646-659.

2. Ponikowski P, et al. Heart failure: preventing disease and death worldwide. ESC Heart Fail. 2014,1135.

3. Abu-Sawwa R, Dunba SB, Quyyumi AA, Sattler ELP. Nutrition intervention in heart failure: should consumption of the DASH eating pattern be recommended to improve outcomes? Heart Fail Rev. 2019,24:565-573.

4. Seidler A, et al. Aircraft, road and railway traffic noise as risk factors for heart failure and hypertensive heart disease-A case-control study based on secondary data. Int J Hyg Environ Health. 2016,219(8):749-758.

5. Hershberger RE, et al. Genetic Evaluation of Cardiomyopathy-A Heart Failure Society of America Practice Guideline. J Card Fail. 2018,24(5):281-302.

6. Kazmierczak K, Yuan CC, Liang JS, Huang WR, Rojas A, Szczesna-Cordary D. Remodeling of the heart in hypertrophy in animal models with myosin essential light chain mutations. Front Physiol. 2014,22(5):353.

7. Kayvanpour E, et al. Genotype-phenotype associations in dilated cardiomyopathy: meta-analysis on more than 8000 individuals. Clin Res Cardiol. 2017,106(2):127-139.

8. Yang QL, et al. Novel phenotype-genotype correlations of hypertrophic cardiomyopathy caused by mutation in a-actin and myosin-binding protein genes in three unrelated Chinese families. $\mathrm{J}$ Cardiol. 2019,73(5):438-444.

9. Feldman D, Carnes C, Abraham WT, Bristow MR. Mechanisms of Disease: $\beta$-adrenergic receptorsalterations in signal transduction and pharmacogenomics in heart failure. Nat Rev Cardiol. 2005,2(9):475-483.

10. Vlahos C, McDowell S, Clerk A. Kinases as therapeutic targets for heart failure. Nat Rev Drug Discov. 2003,2(2):99-113.

11. Hedayat M, Mahmoudi MJ, Rose NR, Rezaei N. Proinflammatory cytokines in heart failure: doubleedged swords. Heart Fail Rev. 2010,15(6):543-562.

12. Yu Y, et al. Brain TACE (Tumor Necrosis Factor-a-Converting Enzyme) Contributes to Sympathetic Excitation in Heart Failure Rats. Hypertension. 2019,74(1):63-72.

13. Stanciu AE. Cytokines in heart failure. Adv Clin Chem. 2019,93:63-113.

14. Rabbani B, Tekin M, Mahdieh N. The promise of whole-exome sequencing in medical genetics. J Hum Genet. 2014,59(1):5-15. 
15. Goodwin S, McPherson JD, McCombie WR. Coming of age: ten years of next-generation sequencing technologies. Nat Rev Genet. 2016,17(6):333-351.

16. Pertea M, Pertea GM, Antonescu CM, Chang TC, Mendell JT, Salzberg SL. StringTie enables improved reconstruction of a transcriptome from RNA-seq reads. Nat Biotechnol. 2015,33(3):290-295.

17. Al-Ayoubi AM, Zheng H, Liu YY, Bai T, Eblen ST. Mitogen-activated protein kinase phosphorylation of splicing factor 45 (SPF45) regulates SPF45 alternative splicing site utilization, proliferation, and cell adhesion. Mol Cell Biol. 2012,32(14):2880-2893.

18. Meyer-Roxlau S, et al. Differential regulation of protein phosphatase 1 (PP1) isoforms in human heart failure and atrial fibrillation. Basic Res Cardiol. 2017,112(4):43.

19. Wang $S$, et al. $\beta$-adrenergic activation may promote myosin light chain kinase degradation through calpain in pressure overload-induced cardiac hypertrophy: $\beta$-adrenergic activation results in MLCK degradation. Biomed Pharmacother. 2020,129:110438.

20. Li YJ, et al. Heart Failure-Related Hyperphosphorylation in the Cardiac Troponin I C Terminus Has Divergent Effects on Cardiac Function In Vivo. Circ Heart Fail. 2017,10(9):e003850.

21. Dong XT, et al. Augmented phosphorylation of cardiac troponin I in hypertensive heart failure. J Biol Chem. 2012,287(2):848-857.

22. Stathopoulou K, et al. S-glutathiolation impairs phosphoregulation and function of cardiac myosinbinding protein C in human heart failure. FASEB J. 2016,30(5):1849-1864.

23. Dridi H, Kushnir A, Zalk R, Yuan Q, Melville Z, Marks AR. Reply to 'Mechanisms of ryanodine receptor 2 dysfunction in heart failure'. Nat Rev Cardiol. 2020.

24. Tomohiro $Y$, et al. p38 Mitogen-activated protein kinase regulates chamber-specific perinatal growth in heart The Journal of Clinical Investigation, J Clin Invest. 2020,130(10): 135859.

25. Dick SA, Epelman S. Chronic Heart Failure and Inflammation What Do We Really Know? Circ Res. 2016,119(1):159-176.

26. Mann DL. Innate immunity and the failing heart: the cytokine hypothesis revisited. Circ Res. 2015,116(7):1254-1268.

27. Pugliese NR, et al. Persistent congestion, renal dysfunction and inflammatory cytokines in acute heart failure: a prognosis study. J Cardiovasc Med. 2020,21(7):494-502.

28. LeWinter MM, Granzier HL. Cardiac Titin and Heart Disease. J Cardiovasc Pharmacol. 2014,63(3):207-212.

29. Franssen C, Miqueo AG. The role of titin and extracellular matrix remodelling in heart failure with preserved ejection fraction. Neth Heart J. 2016,24(4):259-267.

30. Marston S. Obscurin variants and inherited cardiomyopathies. Biophys Rev. 2017,9(3):239-243.

31. Raghow R. An 'Omics' Perspective on Cardiomyopathies and Heart Failure. Trends Mol Med. 2016,22(9):813-827.

32. Randazzo D, Pierantozzi E, Rossi D, Sorrentino V. The potential of obscurin as a therapeutic target in muscle disorders. Expert Opin Ther Targets. 2017,21(9):897-910. 
33. Salcan S, et al. Elastic titin properties and protein quality control in the aging heart. Biochim Biophys Acta Mol Cell Res. 2020,1867(3):118532.

34. Koser F, Loescher C, Linke WA. Posttranslational modifications of titin from cardiac muscle: how, where, and what for? FEBS J. 2019,286(12):2240-2260.

35. Negroni A, Pierdomenico M, Cucchiara S, Stronatl L. NOD2 and inflammation: current insights. J Inflamm Res. 2018,11:49-60.

36. Turkowski KL, Tester DJ, Bos JM, Haugaa KH, Ackerman MJ. Whole exome sequencing with genomic triangulation implicates $\mathrm{CDH}$-encoded $\mathrm{N}$-cadherin as a novel pathogenic substrate for arrhythmogenic cardiomyopathy. Congenit Heart. 2017, 12(2):226-235.

37. Togawa N, Miyaji T, Izawa S, Omote H, Moriyama Y. A Na+-phosphate cotransporter homologue (SLC17A4 protein) is an intestinal organic anion exporter. Am J Physiol Cell Physiol. 2012,302(11):C1652-1660.

38. Bresnick AR. S100 proteins as therapeutic targets. Biophys Rev. 2018,10(6):1617-1629.

39. Ma LP, Haugen E, Ikemoto M, Fujita M, Terasaki F, Fu M. S100A8/A9 complex as a new biomarker in prediction of mortality in elderly patients with severe heart failure. Int J Cardiol. 2012,155(1):26-32.

40. He YY, et al. Usefulness of S100A12 as a prognostic biomarker for adverse events in patients with heart failure. Clin Biochem. 2015,48(4-5): 329-333.

41. Hennecke S, et al. Prevalence of the Prefoldin Subunit 5 Gene Deletion in Canine Mammary Tumors. PLoS One. 2015,10(7):e0131280.

\section{Tables}

Due to technical limitations, tables are only available as a download in the Supplemental Files section.

\section{Figures}



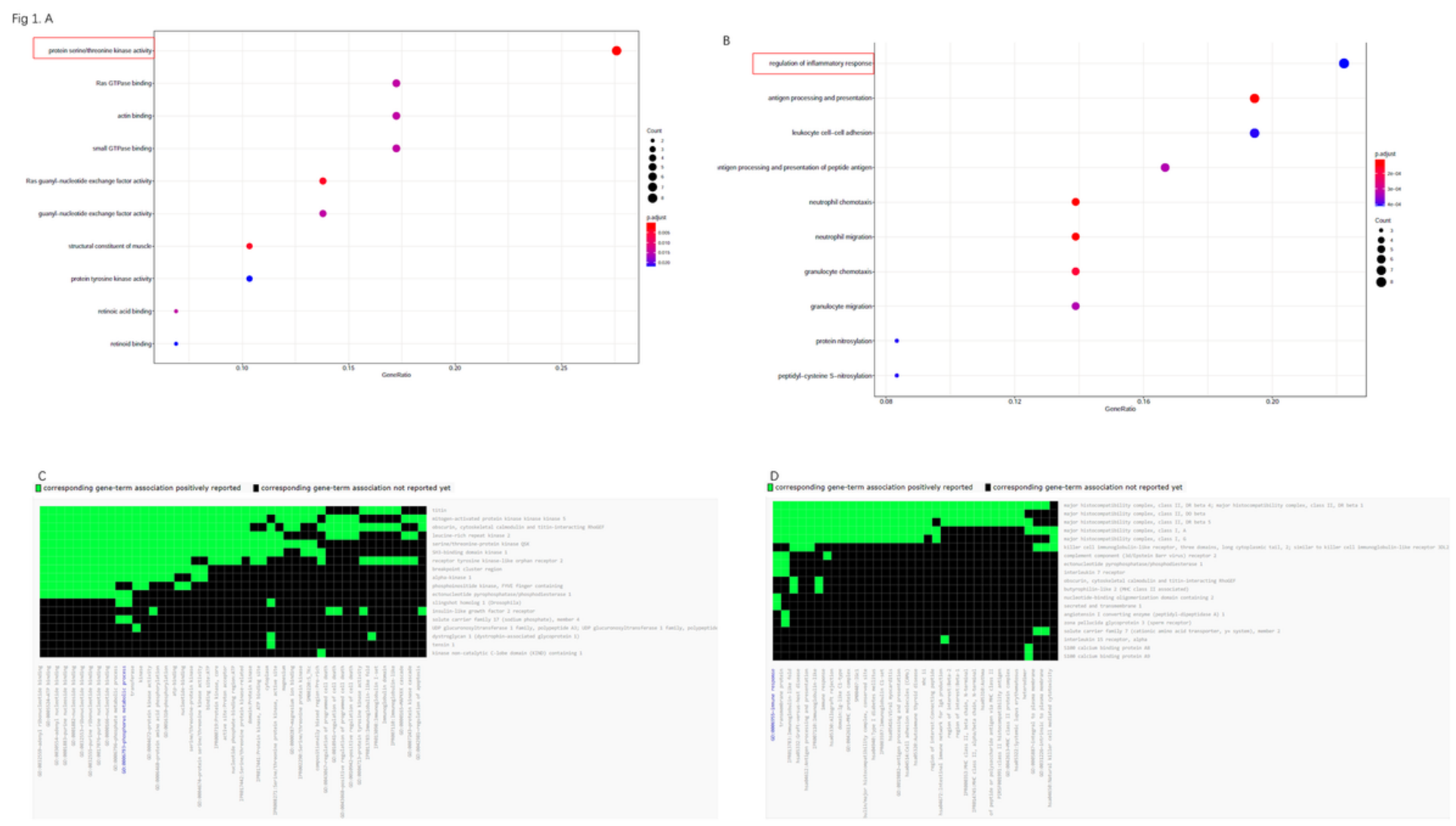

\section{Figure 1}

Bioinformatics analysis of the genes exhibiting mutations in patients with heart failure as detected by whole exome sequencing. (A, B) Biological processes associated with the mutated genes. (C) Gene ontology (GO) analysis of the mutated genes related to phosphorylation processes. (D) GO analysis of the mutated genes related to the immune response. 

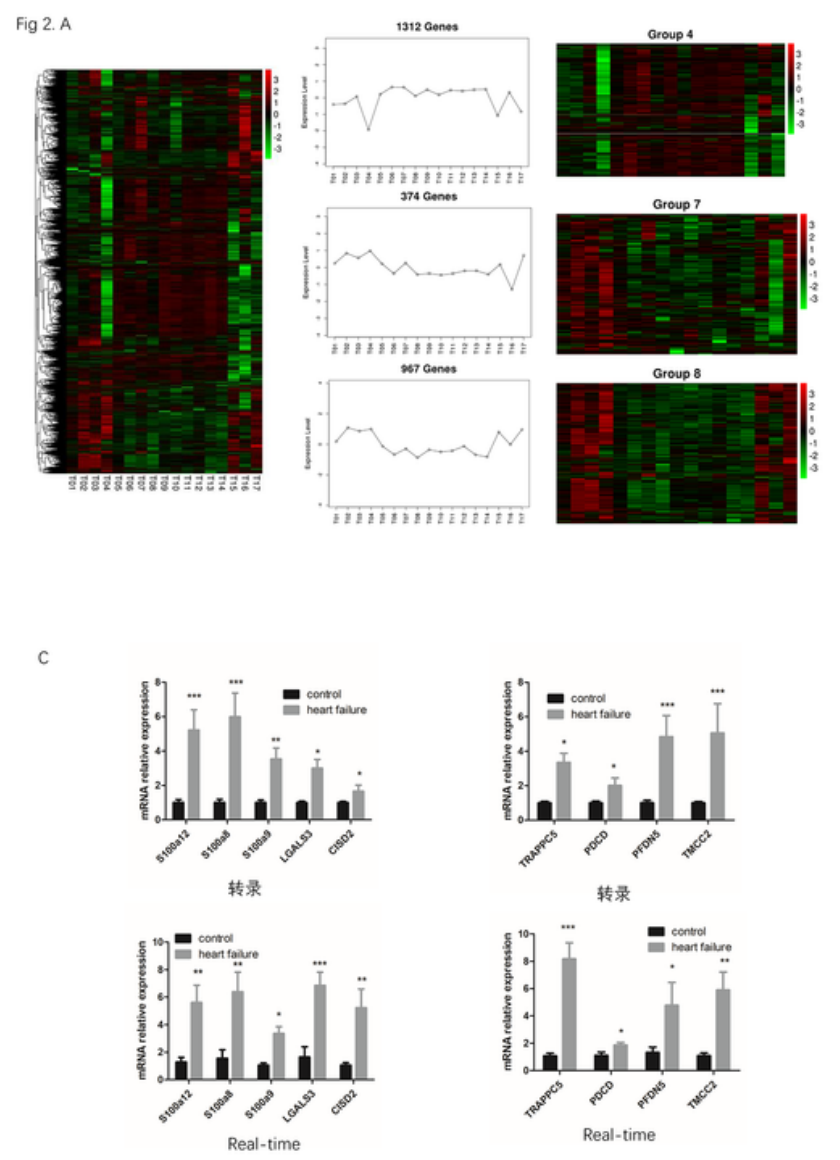

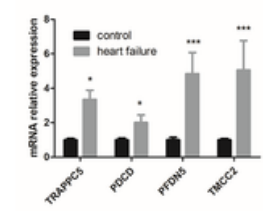

转录

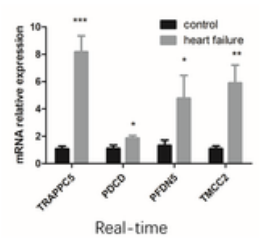

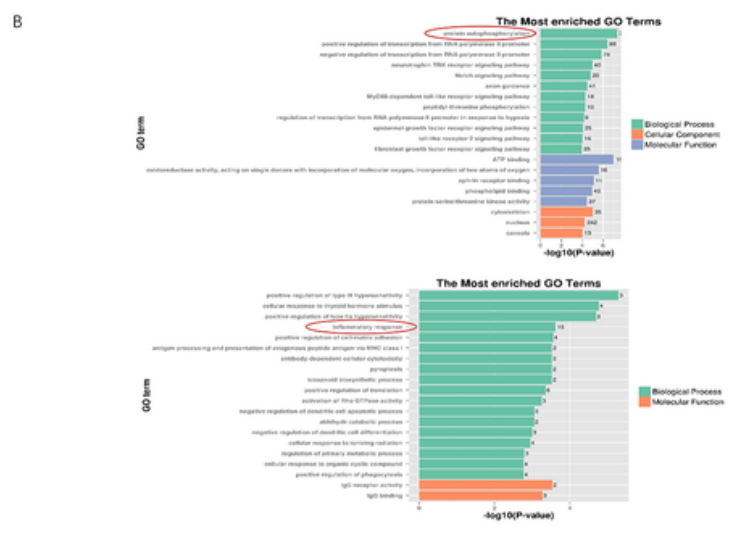

B

D

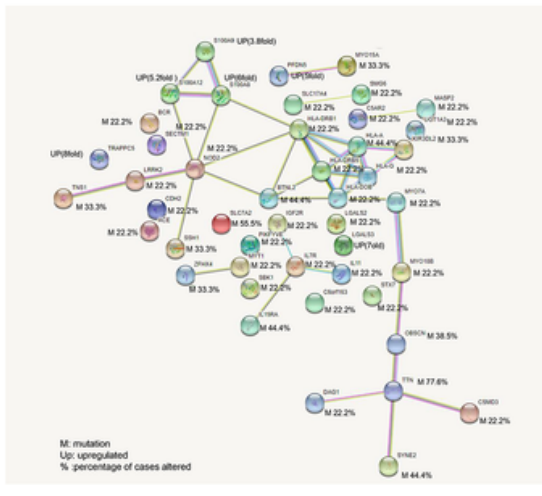

\section{Figure 2}

Bioinformatics analysis of the differentially expressed genes (DEGs) in patients with heart failure (HF) as detected by using RNA-Seq. (A) Co-expression analysis of the DEGs. Red color indicates upregulated genes and green color indicates downregulated genes, the number of up- and down-regulated genes in patients with HF and the clusters of genes enriched and decreased in HF are shown. (B) Biological processes associated with altered genes. (C) Validation of selected DEGs from RNA-Seq analysis using quantitative real-time PCR (qPCR). The first vertical column shows the normalized fold-changes for RNASeq and qPCR of five inflammation-related genes (S100A12, S100A8, S100A9, LGGALS3, and CISD2). The second vertical column shows the normalized fold-changes for RNA-Seq and qPCR of four stressrelated genes (TRAPPC5, PDCD, PFDN5, and TMCC2). (D) The dysregulated signaling pathway. Genes with mutations or copy number changes are listed, and relationships across them are shown by a connection diagram. For each altered gene, the percentage of cases with this alteration is indicated, and for significantly upregulated genes, the average fold-changes are shown.

\section{Supplementary Files}

This is a list of supplementary files associated with this preprint. Click to download.

- table.pdf 
- supplytable.docx

- figure.pdf 